Europe's Journal of Psychology 3/2009, pp. 96-104

www.ejop.org

\title{
An Application of Attribution Theory to Clinical Judgment
}

\author{
Jennifer Murray \\ Mary E. Thomson \\ Glasgow Caledonian University
}

\begin{abstract}
The current article presents an application of attribution theory to clinical judgment, with a focus on the theory's application to clinical judgments of violence risk assessment. While attribution theory has been applied to many diverse fields of human behavior, a comparatively limited level of research and discussion has been raised regarding its application to the very relevant and practical study of clinical decision making (Elbogen, 2002). The current article argues that is not only important for practicing clinicians to understand the way in which their client attributes causality to their behaviour in order to improve upon the effectiveness of therapeutic interventions, but also emphasizes the importance of recognizing and understanding the way in which the clinician attributes causality to the clients behaviour, and how this may affect the development of suitable therapeutic interventions and risk management plans. Through better understanding the effects of attribution on clinical judgments of violence risk assessment, it is argued that improvements to the effectiveness of clinical judgments in violence risk assessment may be possible.
\end{abstract}

Keywords: Attribution, Clinical Judgment, Violence Risk Assessment.

The study of attribution within a clinical decision making setting is both relevant and practical. Elbogen (2002) highlights the issue of relevance in researching a process that is so often described as flawed (i.e., clinical judgment), and suggests that only through further understanding the process, can improvements in researching and practicing the process be accomplished. The present article therefore aims to discuss the relevance and importance of researching the effects of attribution in clinical decision making. Of key interest within the present article is the effects of attribution on the formation of judgments and decision making in violence risk 
assessment. Attribution has received great attention within psychological research since its introduction by Heider (1958), having been applied to a large and varied number of areas. For example, research relating to attribution has been applied to such diverse areas as: motivations in education, personality, organizational and clinical psychology (Weiner, 1985 in Forsterling, 1988).

Heider (1958) proposed that all individuals act as 'naïve scientists' when navigating their environments, both physically and socially, and that they therefore act not only to interpret the physical aspects of their environment, but also the underlying causal properties. Based on this interpretation, Heider (1958) referred to individuals as causal analysts. With regards to person perception in causal analysis, the perceivers manifestation of the object's (the 'target' of the perceivers attention) personality and psychological processes are largely based on observed behavior, but may also be ascertained through sources such as communication with social sources out with the object of perception. It is from this information that a perceptual construction of an individual is created. It must also be noted, however, that the perceiver's own mindset (i.e., the way in which the central processes within the brain interact with the external information presented) acts to determine the way in which the raw material gathered is organized, thus interpreted, by the perceiver.

In accordance with Heider's (1958) theory of interpersonal relations, it can therefore be considered that an individual can infer the characteristics of a person through their perception of that person. Perceptions may be obscure in nature (e.g., an individual can infer another's dissatisfaction but not know how they came to obtain this knowledge) or may be based on more obvious factors (e.g., ascertaining that an individual is displeased based on direct verbal communication to this effect). With this in mind, one must be aware of the dangers of inferring a meaning behind another's actions prematurely. For example, in a risk assessment context, since perception is argued to be a selective process (Plous, 1993), the clinician must be careful not to seek information in line with their initial hypothesis/diagnosis (i.e., confirmatory bias), thus selectively perceiving what they had expected to find.

Heider (1958) further discussed the constancy phenomenon in social perception. The constancy phenomenon describes the expectation of constancy in a perceived object in terms of size, shape, and so on. In the case of person perception this can be applied to aspects such as mood and personality, despite the effects of changing conditions.

Hammond (1955) previously discussed the effects of constancy on person perception in relation to the diagnostic procedures associated with clinical 
judgment. Hammond (1955) asserted that while anger when displayed by another is an observable state, often an individual cannot communicate the reasoning behind how they arrived at this judgment. This is highly relevant in terms of transparency in the clinical judgment process. While the clinician aims to discover the motivations behind patient, client or offender behaviour, several observable behaviours may be apparent in addition to the more subtle internal cues to behavior, as discussed earlier. Heider (1958) suggests that in these 'observer-object' circumstances, the perception of the object and the key observed features remain relatively constant. Furthermore, the impressions formed of another person are said to be based largely on dispositional characteristics, i.e., those that are perceived to be largely consistent in nature. Thus, an individual's behavior may be judged based on a characteristic that is perceived by the observer to be consistent, regardless of inconsistencies in behaviour or circumstances. It is this perceived consistency that aids the clinician in the assessment of risk and prediction of future behaviour. In line with Heider's (1958) theory, the direct impressions formed of another person remain relatively constant over time, despite the level of accuracy/inaccuracy associated with this impression, thus aiding the judgments made relating to predictions of future behaviour.

In order to assess the extent to which constancy may affect clinical judgment, the way in which individuals attribute underlying causation to an individual's behaviour must be understood.

In general, theories of attribution differentiate between causal factors, both internal and external to an individual's behavior (Forsterling, 1988). That is, where an internal attribution is applied to behavior, the individual is considered to be in control or responsible for the outcome. Where an external attribution is applied, a situational or environmental factor out with the individual's control is considered to be responsible for the outcome. For example, if, in a criminal-behaviour context, an internal attribution is applied to an individual, Mr. X, who has committed a theft, one may attribute the cause as something relating to or reflecting Mr. X's personality, thus a 'thrill-seeking' or 'bad person' view of Mr. X may result. If, however, an external attribution is applied, Mr. X may be seen to be in great need of that which he stole, thus he acted out of environmental necessity, and it was therefore his situation that drove him to behave in the way that he did.

In 1967, Harold Kelley systematically developed attribution theory, drawing upon the work of Heider (1958) and Jones and Davis (1965). Kelley (1967) proposed that individuals explain behavior in terms of the person (something about the person caused the behaviour), the entity (a constant of the situation was the cause of the behaviour), or the time (something relating to a specific occasion caused the 
behaviour). It can be seen that the person in this model can be tied to the originally proposed internal attribution of behaviour, and that the entity and time can be related to the originally proposed external attribution of behaviour.

In addition, Kelley (1967) proposed the concept of consistency in judgments of causality, i.e., is an individual's behaviour and/or aspect of the situation consistently present. According to this concept, the way in which we attribute causality to an individual's actions (i.e., was a behavior caused by the person, the entity, or the time) will depend on the perceived consistency of the action/situation, in addition to the distinctiveness of the behavior across other situations and stimuli and the similarity of the action to usual response or social norm. Weiner, Frieze, Kukla, Reed, Rest and Rosenbaum (1971) furthered this concept, discussing stable (constant, unchanging) and variable (changeable over time and situations) causes to behavior, with Rosenbaum (1972) expanding this concept by discussing the importance of differing intentional versus unintentional behaviours. To illustrate this concept in terms of criminal behaviour, relating back to the previous example of Mr. X, the observer will not only consider whether the theft behavior was caused by factors internal or external to Mr. X, as discussed previously, but, in the case of external causality, according to Kelley (1967), will also consider whether there is a constant in the environment (the entity) causing the behavior or if there was a specific trigger on that particular occasion (the time) that has caused the behavior. Prior to finalizing their judgment, the observer will then, in accordance with Weiner et al. (1971) and Rosenbaum (1972), consider whether Mr. X's actions were stable or changeable and whether they were intentional or unintentional.

These causal dimensions are not static, but instead are changeable in meaning across different situations and across different individuals' perceptions of the properties of the cause. Forsterling (1988) illustrated this with the example of failure attributed to moods. He suggested that if a failure is attributed to poor mood, and the mood is believed to be an enduring trait, the individual's response to this will be similar to that triggered by other stable failure attributions, common with enduring internal attributions of causality. If, however, the poor mood is considered to be a temporary state, the individual's response will be similar to that brought about by external attributions. It can therefore be seen that while attributions of causality and the proposed causal dimensions certainly have empirical evidence to support their existence, their definitions and the perceived properties contributing to their formation are changeable from person to person.

While there are only a relatively small number of studies explicitly investigating the effects of attribution on the process of violence risk assessment, research of this 
nature would appear to be of great importance within clinical practice. In 1988, Forsterling presented an application of attribution theory to clinical psychology. This application centred around issues relating to the use of psychotherapy and behavioural modification within clinical psychology. The author proposed that in diagnostic and theoretical interventions, a clinician may benefit through applying attributional analysis. That is, through identifying the way in which an individual client ascribes meaning to their behaviours or emotional status, a clinician can then develop tailored therapeutic interventions which address not only the individual's needs but that also appeals to their specific belief system, ultimately improving the success of the treatment.

However, attribution is not only applicable to the therapeutic aspects of clinical settings, but can also be applied to the judgments and decisions made by clinicians. Just as it is important for the clinician to identify their clients' perceptions of causality, it would also be of great benefit for the clinician to be aware of their own decision making biases. Chen, Froehle and Morran (1997) have shown that trainee counselors who are made aware of their use of biases through training sessions designed to instruct in attribution processes and practice empathetic perspective display significantly lower dispositional bias (i.e., blamed the cause of the presenting problems less on internal factors) than those not receiving the training. In light of the link between the apparent benefits of applying attribution theory within clinical practice, and the evidence to suggest that attributional errors can be effectively reduced in a diagnostic setting, albeit not strictly clinical in nature, a background to attribution within a clinical judgment setting shall now be discussed.

Plous (1993) discussed the impact of attribution on the treatment recommendations made by psychotherapists, suggesting that where a clinician attributes the cause of client behavior to be due to situational factors or due to the clients own disposition, treatment recommendations may differ. Plous (1993) suggested that when a clinician ascribes situational (external) causality, that greater effort is made to alter a client's circumstances. However, where a client's disposition (internal) is judged to be the cause of behaviour, greater effort is placed on altering aspects of the client. While this in itself may not appear to be necessarily of negative consequence, Plous and Zimbardo (1986) reported low levels of reliability in causal explanations across various types of therapy orientations, demonstrating clear implications for client treatment. That is, depending on the orientation of their therapist, the root of a client's problems or behaviours may be considered to be internally or externally based, and so the type of treatment that they would receive would not be equally driven across different types of therapy. 
In discussing the internal versus external perceptions of behavioural cause, there are clear implications for the field of violence risk assessment pertaining to offender behaviour. That is, in order for effective treatment planning and risk management to occur, the causal factors driving the individual's problem(s) must be identified. If these factors are identified as internal, or controllable, a different reaction to the individual may be evoked than if the factors were seen as external. Weiner's (1986) attribution-emotion-action theory proposed that where a negative experience is attributed to internal factors, negative emotions, such as blame, will ensue. However, when these negative experiences are attributed to factors external to the individual, more sympathetic affective responses will ensue. In addition, the way in which cause is attributed can effect expectations for future decision making and behavior accuracy. Curtis (1994) conducted research investigating the inter-professional role conflict experienced in a healthcare setting. Participants were asked to report the perceived causes (attributions) for failure (where the individual had compromised on a decision) and success (where the individual had supported their argument) in terms of their perceived best professional judgment. As could be predicted, success was attributed to internal, stable factors and failure was attributed to external, uncontrollable factors. Curtis (1994) found that attributing cause to external, uncontrollable factors was strongly related to future expectations of failure.

Once again, clear implications for violence risk assessment can be drawn in terms of offender management, from both the perspective of the clinician and the offender. In relation to the former, should the clinician ascribe an internal cause to the offender's negative life experience or behavior, Weiner's (1986) attribution-emotionaction theory would suggest that the clinician would experience blame-type emotions towards the offender, which may in turn affect the judgments made about the offender's behavior. If, in line with Curtis' (1994) findings, this internal causality is deemed to be an enduring, stable feature of the offender, the treatment options and risk management plan suggested may be different from those recommended had the behavior been attributed to an external, unstable cause. This may, of course, be suited to the particular treatment/risk management plan being developed; for instance, in cases involving psychopathy this could be a very important determination of treatment/risk management requirements. However, should internal/external causality be prematurely or incorrectly placed, this would have clear and perhaps serious implications for the treatment of the offender.

As mentioned, the attribution of causality may not only have implications for clinical judgment in a violence risk assessment context, but also for offender judgment. Should the offender ascribe an external cause to their negative life experience or behavior, Weiner's (1986) attribution-emotion-action theory would suggest that the 
offender will develop emotions to cope with their situation, such as sympathy and pity for their own circumstances; emotions protecting the individual from selfblaming. If, in line with Curtis' (1994) findings, this external causality is deemed to be an enduring, stable feature of the offender, the offender may come to expect failure in terms of repeating unwanted behaviours that they perceive to be out with their control. This in turn may lead to a sustained, or indeed increased, incidence of undesirable behaviour, with the offender over time accepting and explaining their negative actions in terms of an external influence driving them or causing them to act in such a way, effectively removing blame from their person.

This mirrored conception of internal/external causal attribution between the clinician and the offender falls in line with Jones and Nisbett's (1972) work on actor-observer differences in causal explanations for behaviour. That is, the tendency for actors (the offender, in this case) to attribute the cause of their actions to situational factors, external to them, while observers (in this case the clinician) to attribute cause to the same action to stable, internal factors.

While the key focus of the present review has been on the effects of attribution on clinical judgments in violence risk assessment, it is fully recognized that judgments and decision making in this field is almost certainly affected by other decision making heuristics and biases not discussed. It would, however, be impossible to address properly all of the possible biases and interactions therein which may affect clinical decision making within the scope of this article. Based on the theoretical arguments and links presented in the current article, it can be concluded that a greater level of empirical research investigating the effects of causal attributions on clinical judgment is required in order to not only expand on current decision making knowledge in this context, but also to inform and improve the practice of clinical decision making in violence risk assessment.

\section{References}

Chen, M., Froehle, T., \& Morran, K. (1997). Deconstructing dispositional bias in clinical inference: Two interventions. Journal of Counseling and Development, 76, 74-81.

Curtis, K. A. (1994). Attributional analysis of interprofessional role conflict. Social Science \& Medicine, 39(2), 255-263.

Elbogen, E. B. (2002). The process of violence risk assessment: A review of descriptive research. Aggression and Violent Behaviour, 7, 591-604. 
Forsterling, F. (1988). Attribution theory in clinical psychology. New York: Wiley.

Hammond, K. R. (1955). Probabilistic functioning and the clinical method. Psychological Review, 62, 255-262.

Heider, F. (1958). The psychology of interpersonal relations. New York: Wiley.

Jones, E. E., \& Davis, K. E. (1965). From acts to dispositions: the attribution process in person perception. In L. Berkowitz (Ed.), Advances in experimental psychology (Vol.2, pp.219-266). New York: Academic Press.

Jones, E. E., \& Nisbett, R. E. (1972). The actor and the observer: Divergent perceptions of the causes of behavior. In E. E. Jones, D. E. Kanhouse, H. H. Kelley, R. E. Nisbett, S. Valins, \& B. Weiner (Eds.), Attribution: Perceiving the causes of behavior (pp.79-94). Morristown, NJ: General Learning Press.

Kelley, H. H. (1967). Attribution theory in social psychology. In D. Levine (Ed.), Nebraska symposium on motivation (pp.192-238). Lincoln: University of Nebraska Press.

Plous, S. (1993). The psychology of judgment and decision making. New York: McGrawHill.

Plous, S., \& Zimbardo, P. G. (1986). Attributional biases among clinicians: A comparison of psychoanalysts and behavior therapists. Journal of Consulting and Clinical Psychology, 54, 568-570.

Rosenbaum, R. M. (1972). A dimensional analysis of the perceived causes of success and failure. Unpublished Dissertation. University of California, Los Angeles.

Weiner, B., Frieze, I. H., Kukla, A., Reed, L., Rest, S., \& Rosenbaum, R. M. (1971). Perceiving the causes of success and failure. New York: General Learning Press.

Weiner, B. (1985). Foreword. In F. Forsterling (Ed.), Attribution theory in clinical psychology (pp.ix-xi). New York: Wiley.

Weiner, B. (1986). An attributional theory of motivation and emotion. New York: Springerverlag. 
About the authors:

Jennifer Murray

Jennifer Murray is currently a PhD student in the Division of Psychology, Glasgow Caledonian University. Her thesis is investigating the effects of attribution on clinical judgments of violence risk assessment, at various stages of the violence risk assessment process.

*Requests for reprints should be addressed to Jennifer Murray, Department of Psychology, Glasgow Caledonian University, 70 Cowcaddens Road, Glasgow, G4 OPP, Scotland, UK (e-mail: jennifer.murray@gcal.ac.uk; tel: 0141552 7990).

Mary E. Thomson

Dr Mary E Thomson is a Reader of Decision Science from the Division of Psychology, Glasgow Caledonian University who has published in various books and journals, including Risk Analysis, Decision Support Systems, the International Journal of Forecasting, the European Journal of Operational Research and the Journal of Behavioral Decision Making. 